\title{
DUPLICATION OF CHROMOSOME 16p13.11-p12.3 WITH DIFFERENT EXPRESSIONS IN THE SAME FAMILY
}

\author{
Pop-Jordanova $\mathrm{N}^{1, *}$, Zorcec $\mathrm{T}^{2}$, Sukarova-Angelovska $\mathrm{E}^{2,3}$ \\ *Corresponding Author: Professor Nadica Pop-Jardonica, Department of Medicine, Research Centre \\ for Genetic Engineering and Biotechnology “Georgi D. Efremov," Macedonian Academy of Sciences \\ and Arts, Bul. Krste Misirkov 2, 1000 Skopje, Republic of North Macedonia. Tel.: +389-2-32-35-400. \\ Fax:+389-2-32-35-423. E-mail: popjordanova.nadica@gmail.com
}

\begin{abstract}
The knowledge about genetic involvement in neurodevelopmental disorders, and especially in autism, is currently rising. To date, more than 100 gene mutations related to autistic syndromes have been described. Some disorders that affect multiple family members are caused by gene mutations, which can be inherited. Recently, array comparative genomic hybridization $(\mathrm{aCGH})$ has identified sub microscopic deletions and duplications as a common cause of mental retardation and autism. In this article we report the occurrence of the same genetic finding (chromosome 16p13.11-p12.3 duplication) in a family with four small children, where two older siblings manifested a global neurodevelopmental delay associated with an autism spectrum disorder (ASD), but younger twin brothers with the same mutation, have typical development. Genetic analysis showed that the chromosomal duplication was inherited from the father, in which phenotype and functioning are quite typical. As is known, the duplication can pass from parents to children. The 16p13.11 micro duplication has been implicated in several neurodevelopmental and behavioral disorders and is characterized by variable expressivity and incomplete penetrance.
\end{abstract}

Keywords: Autism; Family; Genetics; Neurodevelopmental delay.

\footnotetext{
Department of Medicine, Research Centre for Genetic Engineering and Biotechnology "Georgi D. Efremov," Macedonian Academy of Sciences and Arts, Skopje, Republic of North Macedonia

${ }^{2}$ Department of Psychophysiology, University Children's Hospital, Faculty of Medicine, University "Cyril and Methodius," Skopje, Republic of North Macedonia

${ }^{3}$ Department of Endocronology and Genetics, University Clinic for Children's Diseases, Medical Faculty, University Sv. Kiril i Metodij, Skopje, Republic of North Macedonia
}

\section{INTRODUCTION}

Autism spectrum disorder (ASD) or autism, is a condition that appears early in childhood development, varies in severity, and is characterized by impaired social skills, communication problems and repetitive behaviors. These difficulties can interfere with affected individuals' ability to function in social, academic and employment settings [1]. Children with ASD also have an increased risk of psychiatric difficulties such as anxiety, depression, obsessive compulsive disorder and eating disorders, later in the life. In the last decade the prevalence of this condition has been increased, and it is being referred to as an epidemic. The etiology is assumed to be a combination of genetic and environmental factors. However, for the further development of the child, it is very important to make the diagnosis as early as possible and to include different available therapies. Prenatal diagnosis for autism is unfortunately not yet possible.

Autism and intellectual impairment are often associated, suggesting that these conditions are etiologically related. Recently, array comparative genomic hybridization (aCGH) has identified sub microscopic deletions and duplications as a common cause of intellectual impairment, prompting us to also search for such genomic imbalances in autism [2].

Currently, the knowledge about genetic involvement in neurodevelopmental disorders is rising. To date, more than 100 gene mutations related to autistic syndromes have been described. Some disorders that affect multiple family members are caused by gene mutations, which can be inherited. This situation might be described as "running in the family." In this context, genetic analysis is indicated, especially in the case when two or more members of the same family have similar phenotypes. Whole exome sequencing (WES) represents a significant breakthrough in clinical genetics as a powerful tool for etiological discovery in neurodevelopmental disorders. 
In the last decade, microarray-based copy number variation $(\mathrm{CNV})$ analysis has been proved as a particularly useful strategy in the discovery of loci and candidate genes associated with phenotypes characterized by significant deficits in cognitive and adaptive skills during the developmental period, and it is widely used in the clinics with a diagnostic purpose. Research confirmed that CNVs are a common cause of intellectual impairment and/or multiple congenital anomalies. Today, rare $\mathrm{CNVs}$ are recognized as an important cause of various neurodevelopmental disorders, including intellectual impairment and epilepsy. In some cases, a second $\mathrm{CNV}$ may contribute to a more severe clinical presentation [3-5].

The chromosome 16p13.3.11 duplication is a chromosome abnormality that can affect many parts of the body. People with this condition have an extra piece of genetic material (duplication) on chromosome 16 at a location designated p13.3. The symptoms and severity vary from person to person because not everyone with a $16 \mathrm{p} 13.3 .11$ duplication has the same amount of extra DNA. Possible symptoms include developmental delay (DD), speech delay, joint abnormalities, characteristic facial features, attention deficit, autism and underlying health problems such as heart conditions. Most cases are not inherited and occur randomly. Less commonly, the duplication is inherited from a parent [6].

In this study, we report a family occurrence of the same genetic finding (chromosome 16p13.3.11 duplication) where two siblings manifested a global neurodevelopmental delay associated with an autism and the other two (twin) brothers without clinical manifestation of neurodevelopmental issues. Genetic analysis showed that the mutation was inherited from the father.

\section{CASE REPORT}

The proband, MG, female, first came to the University Children's Hospital, Skopje, Republic of North Macedonia (RNM) 4 years ago, at the age of 2.5 years, with suspected developmental delay (DD). Clinical observation confirmed speech and language difficulties (absence of expressive language and poor receptive language), poor nonverbal communication, poor gaze phenomena, not responsive to her own name, very poor social skills and interaction, many stereotyped behaviors, sensory difficulties, poor play skills and poor general knowledge. She was diagnosed as being autistic, according to the criteria for autism described in the Diagnostic and Statistical Manual of Mental Disorders, 5th edition (DSM-5) [1]. She started receiving therapies from the special educators and rehabilitators, speech and language therapists and psychologists such as sensory integration, speech therapy and psychomotor reeducation. At the hospital, she still receives treatments with joint attention, symbolic play, engagement regulation (JASPER) and discrete trial training (DTT). Preschoolbased JASPER intervention in minimally verbal children with autism, is highly recommended and provides good results [7]. Additionally, DTT is a structured technique that breaks down skills into small, "discrete" components. Systematically, the therapist teaches these skills one by one [8]. At some point of the treatment, she received medications such as Risperidone (antipsychotic) and Nootrop (stimulant) due to severe regression after a varicella infection. She is now 6 years and 4 months old. Her expressive language is evaluated as equal to a 5-year-old child, receptive language and nonverbal communication is equal to her peer group. General knowledge is above her age group. There is still presence of stereotyped behavior, restrictive and repetitive interest and activity, and she is still very anxious during social interaction. There is no spontaneous behavior at all; every goal-oriented activity needs to be prompted.

Her younger brother (MIG) first came to the University Children's Hospital 2 years ago, when he was 2 years old. Clinical observation confirmed speech and language difficulties (absence of expressive language and very poor receptive language), very poor nonverbal communication, very poor gaze phenomena, not responsive to his own name, very poor social skills and interaction, many stereotyped behaviors, very poor play skills, very poor general knowledge and frequent meltdowns. He was also diagnosed as being autistic, according to the DSM-5 criteria for autism. Generally, his clinical manifestation of autism was much more severe than in his sister. He was treated in the same manner as his sister. He is now 4 years and 9 months old. His expressive and receptive language is evaluated as equal to a 3-year-old child, his nonverbal communication and general knowledge are equal to his peer group, there is still strong presence of stereotyped behavior, restrictive and repetitive interest and activity, and he is very anxious during social interaction. Meltdowns are now rare.

Both siblings do not have significant dysmorphic features. At the time of obtaining the genetic results of the first two siblings, the mother was 6 months pregnant with twins. The genetic evaluation for this duplication was not available at this time, so the parents decided to go forward with the pregnancy. The twin boys were evaluated soon after delivery, with extensive genetic counseling of the parents. The same genetic duplication was also confirmed in both of the twins. At the moment, the twins are 26 months old. They have been evaluated to have a delay of 6 months in expressive language, but all other developmental milestones have been met. The same duplication was also 
found in the father, who has high intellectual capacities and without any psychiatric disorders. The family tree of this family is presented in Figure 1.

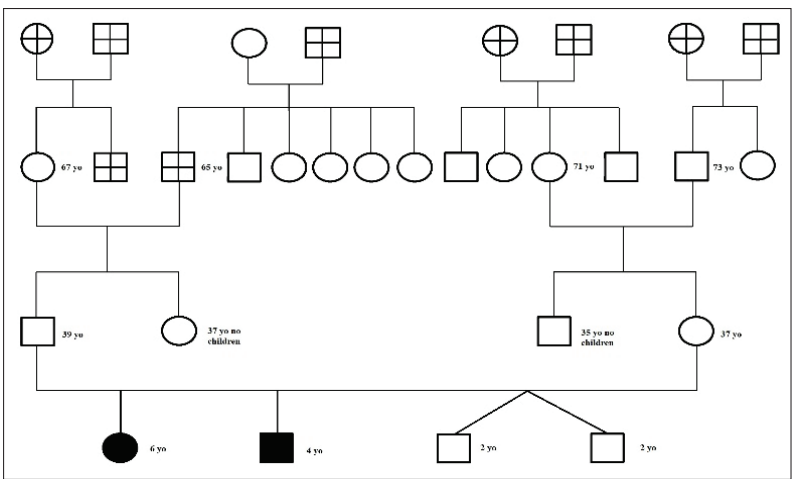

Figure 1. Family tree for the affected family.

Genetic analysis was performed by the team of Research Centre for Genetic Engineering and Biotechnology "Georgi D. Efremov" (ICBIB) at the Macedonian Academy of Sciences and Arts, Skopje, RNM. The procedure was as follows: 1) isolation of DNA with phenolchlorophorm and ethanol precipitation; 2) aCGH with Sure Print G3 CGH 4×180 kb, oligo microarray kit; Agilent Technologies, Santa Clara, CA, USA, mean distance between probes was $13 \mathrm{~kb}$; the analysis of the results used Agilent Technologies genomic Workbench software and database for genomic variants, University of Santa Cruz (UCSC) genome browser, ClinVar database (https://www. clinical genome.org). The obtained result was: ISCN arr cgh 16p.13.11-p12.3 $(15,399,818-18,069,668) \times 3$. The referent sequence: human genome builds 36 (hg 18).

Interpretation. The aCGH of DNA material obtained for the patients showed chromosomic micro duplication of the short arm of the chromosome in the 16p13.11-p12.3 region. The minimal size of the duplication is $2.67 \mathrm{Mb}$. The deleted region encompasses 11 genes: $M V P 17 L, C 16$ orf45, KIAA0430, NDEI, MYH11, C16orf63, ABCC1, ABCC6, NOMO3, LOC339047, XYLTI.

The database information confirms this duplication to be related to neurodevelopmental disorders, cognitive delay, some neuropsychiatric disorders, but also in people with a "normal phenotype." Results obtained for our patients confirmed genetic etiology of neurodevelopmental delay as well as autistic behavior. The same mutation was found in the twins: $\mathrm{I}=16 \mathrm{p} .13 .11-\mathrm{p} 12.3$ (15492317 $18162167) \times 4$ with the minimal size of the duplication of $2.67 \mathrm{Mb}$; II = 16p.13.11-p12.3 (15492317_18162167) $\times 4$ with the same size of the duplication of $2.67 \mathrm{Mb}$. Fortunately, their follow-up showed practically typical development.

\section{DISCUSSION}

Generalized clinical use of chromosomal microarray analysis (CMA) in etiological investigation of neurodevelopmental disorders, has led to the decryption of many new CNVs, such as those in $16 \mathrm{p} 13.11$. Structural variation of the human genome results from genomic rearrangements including deletions, duplications, insertions and inversions. All of these genomic rearrangements, except for inversions, result in $\mathrm{CNV}$ or deviation from the normal number of copies for a given genomic segment. Although the widespread utilization of one such tool, aCGH has led to the discovery of many novel genomic disorders. One such CNV with a yet uncharacterized clinical phenotype is a rearrangement in chromosome $16 \mathrm{p} 13.11$, associated with epilepsy, multiple congenital anomalies and cognitive impairment, while duplications have been implicated in ASDs, intellectual disability and schizophrenia [9,10].

The Medical Genetics Laboratories at Baylor College of Medicine, Houston, TX, USA has performed over 14,000 aCGH for clinical evaluation of subjects with DD, dysmorphic features and/or multiple congenital anomalies from June 2007 to January 2010 [6]. During this period, they identified 56 patients with duplications and 30 patients with deletions of $16 \mathrm{p} 13.11$. Nagamani et al. [6] concluded that the clinical spectrum associated with both duplications and deletions are quite variable and the manifestations are incompletely penetrant, making genetic counseling of such families a challenging prospect. Ramalingam et al. [11] confirmed that the clinical significance of 16p13.11 duplications remains controversial, while frequently detected in patients with DD, intellectual deficiency (ID) or ASD.

Searching in the literature, we found a study by Ullmann et al. [12], who described a 1.5 Mb duplication on chromosome $16 \mathrm{p} 13.1$ that was found by high resolution aCGH in four severe autistic male patients from three unrelated families. In a study by Tropeano et al. [13], there was reported evidence for a male-biased autosomal effect of $16 \mathrm{p} 13.11$ duplications and deletions in a sample of 10,397 individuals with a neurodevelopmental condition, analyzed by whole genome aCGH. The CNVs identified included 28 duplications with sizes ranging from 0.8 to $3.29 \mathrm{Mb}$, and 18 deletions with sizes between 0.02 and 3.26 $\mathrm{Mb}$ [two non allelic homologous recombination (NAHR)mediated]. It was suggested that $\mathrm{CNVs}$ at chromosome $16 \mathrm{p} 13.11$ have been associated with a range of neurodevelopmental disorders including autism, attention deficit hyperactivity disorder (ADHD) intellectual disability and schizophrenia [14].

Quintela et al. [14] described maternally inherited 16p13.11-p12.3 duplication and a de novo 12p12.1 deletion affecting $S O X 5$ in a male patient with global DD, disruptive 
and obsessive behaviors and minor dysmorphic features. It was noted that the $16 \mathrm{p} 13.11$ micro duplication has been implicated in several neurodevelopmental and behavioral disorders and is characterized by variable expressivity and incomplete penetrance [14].

In this context, Pinto et al. [15] published exome sequencing analysis in a pair of monozygotic twins that reevaluates the genetics behind their intellectual disability and reveals a $C H D 2$ mutation in two monozygotic twins. An aCGH analysis revealed two maternally inherited duplications on chromosomes 8p22 and 16p13.11 in both twins. Their study underlines the importance of considering a $C H D 2$ gene involvement in children with intellectual disability and ASD [15].

Srivastava et al. [16] suggested the high diagnostic yield of WES and supported its use in pediatric neurology practices. This method may also lead to earlier diagnosis, impacting medical management, prognostication and family planning. Therefore, WES serves as a critical tool for the child neurologist [16].

Unlike apparent CNV that are clearly pathogenic, $16 \mathrm{p}$ micro duplication have a wide range of phenotypic variability. Intra familial phenotypic variability of $16 \mathrm{p} 11.2$ duplication syndrome has been described in many reports, ranging from normal to severely affected cognitive impairment $[17,18]$.

Our finding is similar to those described in the literature, having unaffected father and the latter two children, and variable presentation of autism in the first two children. The reason for such variability could be the existence of a recessive mutation in one of the genes on the maternal chromosome, existence of an imprinted gene in the region, or involvement of other modifier genes that contribute the phenotype.

Conclusions. Our case report confirmed the genetic involvement of arr cgh $16 \mathrm{p} .13 .11$ in neurodevelopmental delay combined with autistic behavior in two siblings and the father in the same family. The obtained results are supported in some other studies. The same gene mutation was found in twin brothers, with no significant developmental issues so far.

The clinical spectrum associated with both duplications and deletions are quite variable and the manifestations are incompletely penetrant, making genetic counseling of such families difficult. However, genetic evaluation in the large spectrum of neurodevelopmental disorders is needed.

Declaration of Interest. The authors report no conflicts of interest. The authors alone are responsible for the content and writing of this article.

\section{REFERENCES}

1. American Psychiatric Association: Diagnostic and Statistical Manual of Mental Disorders, 5th ed. Arlington, VA, USA: American Psychiatric Association, 2013.

2. Vulto-van Silfhout AT, Hehir-Kwa JY, van Bon BWM, Schuurs Hoeijmakers JHM, Meader S, et al. Clinical significance of de novo and inherited copy number variation. Hum Mutat. 2013; 34(12): 16791687.

3. Itsara A, Cooper GM, Baker C, Girirajan S, Li J, Absher D, et al. Population analysis of large copy number variants and hotspots of human genetic disease. Am J Hum Genet. 2009; 84(2): 148-161.

4. Hannes FD, Sharp AJ, Mefford HC, de Ravel T, Ruivenkamp CA, Breuning MH, et al. Recurrent reciprocal deletions and duplications of 16p13.11: The deletion is a risk factor for MR/MCA while the duplication may be a rare benign variant. J Med Genet. 2009; 46(4): 223-232.

5. Shinawi M, Liu P, Kang SH, Shen J, Belmont JW, Scott DA, et al. Recurrent reciprocal 16p11.2 rearrangements associated with global developmental delay, behavioural problems, dysmorphism, epilepsy, and abnormal head size. J Med Genet. 2010; 47(5): 332-341.

6. Nagamani SCS, Erez A, Bader P, Lalani SR, Scott DA, Scaglia F, et al. Phenotypic manifestations of copy number variation in chromosome $16 \mathrm{p} 13.11$. Eur J Hum Genet. 2011; 19(3): 280-286.

7. Goods KS, Ishijima E, Chang Ya C, Kasari C. Preschool based JASPER intervention in minimally verbal children with autism: Pilot RCT. J Autism Dev Disord. 2013; 43(5): 1050-1056.

8. Okuno T, Kondelis N. Evaluation of dithiothreitol (DTT) for inactivation of IgM antibodies. J Clin Pathol. 1978; 31(12): 1152-1155.

9. Loureiro S, Almeida J, Café C, Conceição I, Mouga $\mathrm{S}$, Beleza A, et al. Copy number variations in chromosome 16p13.11-The neurodevelopmental clinical spectrum. Curr Pediatr Res. 2017; 21(1): 116-129.

10. Liu JYW, Kasperavičiūtė D, Martinian L, Thom M, Sisodiya SM. Neuropathology of 16p13.11 deletion in epilepsy. PLoS One. 2012; 7(4): e34813.

11. Ramalingam A, Zhou XG, Fiedler SD, Brawner SJ, Joyce JM, Liu H-Y, et al. 16p13.11 duplication is a risk factor for wide spectrum of neuropsychiatric disorders. J Hum Genet. 2011; 56(7): 541-544. 
12. Ullmann R, Turner G, Kirchhoff M, Chen W, Tonge B, Rosenberg C, et al. Array CGH identifies reciprocal $16 \mathrm{p} 13.1$ duplications and deletions that predispose to autism and/or mental retardation. Hum Mutat. 2007; 28(7): 674-682.

13. Tropeano M, Ahn JW, Dobson RJB, Breen G, Rucker J, Dixit A, et al. Male-biased autosomal effect of 16 p13.11 copy number variation in neurodevelopmental disorders. PLoS One. 2013; 8(4): e61365.

14. Quintela I, Barros F, Lago-Leston R, Castro-Gago M, Carracedo A, Eiris J. A maternally inherited 16p13.11-p12.3 duplication concomitant with a de novo SOX5 deletion in a male patient with global developmental delay, disruptive and obsessive behaviors and minor dysmorphic features. Am J Med Genet A. 2015; 167(6): 1315-1322.

15. Pinto AM, Bianciardi L, Mencarelli MA, Imperatore V, Di Marco C, Furini S, et al. Exome sequencing analysis in a pair of monozygotic twins re-evaluates the genetics behind their intellectual disability and reveals a CHD2 mutation. Brain Dev. 2016; 38(6): 590-596.

16. Srivastava S, Cohen JS, Vernon H, Barañano K, McClellan R, Jamal L, et al. Clinical whole exome sequencing in child neurology practice. Ann Neurol. 2014; 76(4): 473-483.

17. Weiss LA, Shen Y, Korn JM, Arking DE, Miller DT, Fossdal R, et al. Association between microdeletion and microduplication at $16 \mathrm{p} 11.2$ and autism. N Engl J Med. 2008; 358(7): 667-675.

18. Sahoo T, Theisen A, Rosenfeld JA, Lamb AN, Ravnan JB, Schultz RA, et al. Copy number variants of schizophrenia susceptibility loci are associated with a spectrum of speech and developmental delays and behavior problems. Genet Med. 2011; 13(10): 868-880. 
